Nuevas perspectivas en la resolución del error médico: National Health Service Resolution y CARe

\title{
New perspectives in resolving medical error: National Health Service Resolution and CARe
}

https://doi.org/10.23938/ASSN.0388

L. Aragonés ${ }^{1}$, A. Hunter ${ }^{2}$, C. Martin-Fumadó ${ }^{1,3}$, J. Arimany-Manso ${ }^{1,3}$

\section{Sr. Editor:}

En 1999, la publicación del informe To err is human: Building a safer health system de la mano del Institute of Medicine de EEUU ${ }^{1}$, pretendía acabar con el silencio en torno al error médico y sus consecuencias sin culpabilizar a los profesionales. Desde entonces, la seguridad clínica, entendida como el conjunto de actuaciones orientadas a eliminar, reducir y mitigar los resultados adversos producidos como consecuencia del proceso de atención a la salud ${ }^{2}$ ha experimentado un notable progreso, desarrollándose medidas organizativas, protocolos de calidad y procesos más seguros. Aun así, la comunicación voluntaria del error médico sigue siendo, en nuestro contexto, un asunto pendiente de resolver ${ }^{3}$.

Los profesionales temen una reclamación judicial, con el consecuente desprestigio personal y una posible inhabilitación profesional. Más allá de las graves consecuencias sobre el profesional que una reclamación puede dar lugar ${ }^{4}$, el temor a una reclamación ante el más mínimo error mientras se ejerce la profesión puede comportar también la práctica de medicina defensiva ${ }^{5}$. Sin embargo, los datos del Servicio de Responsabilidad Profesional del Colegio de Médicos de Barcelona, que gestiona las reclamaciones por supuesta mala praxis médica de los cuatro colegios de médicos provinciales de Cataluña, confirman que es extremadamente infrecuente que exista una inhabilitación profesional, lo que demuestra que se trata, en gran medida, de un temor infundado ${ }^{6}$.

Por otro lado, para conseguir un sistema de salud más seguro y reestablecer la natural alianza entre médico y paciente es fundamental identificar y aprender de los errores $^{7,8}$ mediante el desarrollo de un sistema público de información a nivel nacional, alentando a las organizaciones y profesionales de la salud a desarrollar y participar en dichos sistemas de notificación voluntaria.

Así mismo, una parte esencial en la comunicación del error es la disculpa que debe acompañar a la información del mismo. En
1. Servicio de Responsabilidad Profesional. Área de Praxis. Colegio de Médicos de Barcelona. Consejo de Colegios de Médicos de Catalunya. Barcelona.

2. Management consultant. Former Director of Claims Department. National Health Service Resolution. London.

3. Cátedra de Responsabilidad Profesional Médica y Medicina Legal. Universitat Autònoma de Barcelona. Barcelona.

\section{Correspondencia:}

Carles Martin-Fumadó

Unidad de Medicina Legal

Servicio de Responsabilidad Profesional

Colegio de Médicos de Barcelona

Passeig de la Bonanova, 47

08017 Barcelona

E-mail: carles.martin@comb.cat

Recepción: 09/11/2018

Aceptación definitiva: 04/12/2018 
este sentido, el consenso entre expertos españoles en la materia, con la que estamos absolutamente de acuerdo, recomienda que la comunicación al paciente debe incluir información sobre lo que ha sucedido, cómo ha sucedido, sus posibles consecuencias y secuelas, las medidas tomadas al respecto, así como una disculpa expresada en términos neutros, de forma que el paciente escuche "lo siento" por lo ocurrido". De hecho, el 90\% de los profesionales comparten la idea de que se debe informar a los pacientes de lo sucedido ${ }^{10}$. Por otro lado, también son aspectos relevantes de dicha comunicación el cuándo y quién debe informar, entendiendo que no debe transcurrir mucho tiempo entre el incidente y el momento de la comunicación y que la persona que informa debe valorarse en función de cada caso y no necesariamente debe encargarse la comunicación al profesional más directamente ligado al evento adverso.

De lo que no hay duda es que la comunicación del error, tras un abordaje integral, no debe ser improvisada, sino que debe ser el resultado de un análisis dirigido a la comunicación de dicho error ${ }^{3}$. Tampoco hay duda de la mejora que esta comunicación supone en la relación médico-paciente. Sin embargo, a nivel de organizaciones, una cultura de seguridad clínica poco madura impide disponer de la información necesaria al respecto y a nivel del profesional, existe consenso en la necesidad de adquirir las competencias y habilidades para llevar a cabo una óptima comunicación de los eventos adversos, estimándose necesaria una formación específica durante la formación médica básica ${ }^{3}$.

Tras esta comunicación, se ha señalado a la mediación como el camino ideal hacia la búsqueda de soluciones de una forma sencilla y funcional ${ }^{9}$. Sin embargo, la comunicación de los eventos adversos queda condicionado absolutamente por el marco legal existente en cada país. En otros entornos distintos al nuestro, la comunicación goza del denominado privilegio legal, entendiéndose la misma como un primer paso hacia la reparación y dentro de un marco de seguridad jurídica para el profesional. En España, por el contrario, no solo no exis- te este beneficio sino que la información y disculpa puede derivar en responsabilidad profesional $^{11}$.

En este contexto cabe mencionar dos iniciativas internacionales revolucionarias que han supuesto un salto cualitativo en transparencia (comunicación del error), responsabilidad profesional (reparación del error) y seguridad clínica (aprendizaje de los errores). La primera, del National Health Service (NHS) en el Reino Unido, es de gran trascendencia dado que es obligatoria para todo el sistema de salud. La segunda, de la Alianza de Massachusetts para la Comunicación y Resolución ante un Error Médico (MACRMI) de EEUU, por el contrario, solo compromete a los hospitales adheridos voluntariamente.

A raíz del escándalo del hospital Mid Stafford, el NHS y el General Medical Council apoyaron la regulación en Reino Unido del deber de franqueza o duty of candour (Tabla 1), estableciendo el deber legal, desde 2014, de transparencia y honestidad con el paciente o con sus familiares ante cualquier evento adverso.

El cambio de denominación de la $\mathrm{NHS} \mathrm{Li}$ tigation Authority a NHS Resolution (NSHR) refleja el cambio de paradigma experimentado en la organización, pasando de ser un organismo que tenía como principal misión la defensa del NHS ante las reclamaciones por parte de los usuarios de la sanidad por supuesta mala praxis médica, a un sistema con mayor transparencia y responsabilidad, que pretende mejorar la seguridad clínica aprendiendo de los errores y asegurándose, desde el departamento Safety and Learning, que el aprendizaje esté disponible y se comparta en todo el NHS. Para ello elabora periódicamente recomendaciones generales, como la guía Saying Sorry Guidance $^{12}$, destinada a facilitar la buena relación médico-paciente. Este nuevo sistema busca el consenso con el paciente: se pretende tenerlo más en cuenta en la toma de decisiones intentando menguar las consecuencias de los daños sufridos por este ante una eventual mala praxis.

Asimismo, la aproximación al paciente, mediante las denominadas cartas de disculpa (que no son admisiones de culpa) 
Tabla 1. Deber de franqueza (duty of candour) del National Health Service del Reino Unido

\begin{tabular}{|c|c|}
\hline \multicolumn{2}{|c|}{ Los profesionales de la salud deben: } \\
\hline 1. & $\begin{array}{l}\text { Dar explicaciones al paciente (o cuando corresponda al cuidador, la familia o el representante del } \\
\text { paciente) cuando el tratamiento o asistencia dispensada no ha ido como se esperaba o es suscep- } \\
\text { tible de crear algún tipo de daño. }\end{array}$ \\
\hline 2. & $\begin{array}{l}\text { Pedir disculpas al paciente (o cuando corresponda al cuidador, la familia o el representante del } \\
\text { paciente). }\end{array}$ \\
\hline 3. & Ofrecer un remedio o apoyo para intentar solucionar la situación. \\
\hline 4. & $\begin{array}{l}\text { Dar explicaciones al paciente (o cuando corresponda al cuidador, la familia o el representante del } \\
\text { paciente) acerca de los efectos a corto y a largo plazo que puede llegar a tener el evento adverso } \\
\text { que se haya producido. }\end{array}$ \\
\hline \multicolumn{2}{|r|}{$\begin{array}{l}\text { Los profesionales de la salud también deben estar abiertos y ser honestos con sus colegas, empleadores } \\
\text { y organizaciones, y participar en las revisiones e investigaciones cuando se les solicite. También deben } \\
\text { estar abiertos y ser honestos con sus reguladores, planteando inquietudes cuando sea apropiado. Deben } \\
\text { apoyarse y alentarse mutuamente para ser estar abiertos y ser honestos, y no evitar que alguien exprese } \\
\text { sus inquietudes. }\end{array}$} \\
\hline
\end{tabular}

favorece la mediación como sistema preferente de resolución de conflictos. El NHS ha sido pionero en el uso de este sistema, que permite dar apoyo a los pacientes y sus familiares y facilita la interacción entre ellos, proporcionando un trabajo conjunto para la resolución de incidencias, reclamaciones y quejas. De esta forma se pretenden evitar los gastos innecesarios que supone la litigación judicial. Así, en el periodo 2016-2017, el NHSR recibió 10.686 reclamaciones, resolviéndose hasta un $67,8 \%$ de manera extrajudicial (mediante negociación, celebrando reuniones entre ambas partes o mediante mediación formal como método de resolución alternativa de conflictos). Además, de las reclamaciones que acabaron llegando a los juzgados, menos de un $1 \%$ terminaron en juicio, siendo la sentencia favorable para el NHS ${ }^{13}$ en casi todas ellas.

Por otro lado, el NHS tiene instaurado un esquema de actuación que supone para todos los hospitales la obligación de comunicar al NHSR todos los incidentes relacionados con la maternidad producidos a partir del 1 de abril de 2017. Esta obligación permite investigar las circunstancias en las que se produjo el error, dotar de un mayor apoyo a la familia y compartir el aprendizaje rápidamente. Por el momento, esta obligación solo existe para Obstetricia, pues supone el $50 \%$ de las indemnizaciones por reclamaciones del $\mathrm{NHS}^{14}$. En las demás especialidades, el deber de comunicación del error es con el paciente y es este quien decide si presenta o no la reclamación al NHSR.

Un modelo similar que, como ya ha sido expuesto, se diferencia del anterior en la voluntaria adhesión al programa, es el de la MACRMI ${ }^{15}$, que ha desarrollado el programa CARe (Communication, Apologize, Resolution) que establece los pasos a seguir ante un error médico. Los objetivos del programa son: a) mejorar la comunicación y la transparencia ante un error médico, b) apoyar a pacientes y familias ayudando a lograr una resolución justa de daños médicos, c) apoyar a los médicos en la comunicación de los errores a los pacientes, d) mejorar la seguridad del paciente aprendiendo de los errores y previniendo daños futuros. La comunicación del error debe realizarse inmediatamente, escuchando las preocupaciones del paciente y su familia. A posteriori se investigará la causa del mismo, valorando si podía haber sido evitado, y si fuese el caso, averiguar cómo prevenirlo en un futuro. Se comparte el aprendizaje fruto de la investigación con el paciente y se explican las medidas que se tomarán para que no vuelva a producirse el mismo error. Finalmente, es necesaria una disculpa sincera si a raíz de la investigación se concluye que el error podría haberse evitado. 
De la misma manera que el NHSR, el programa CARe proporciona una alternativa a los pleitos y sus costos innecesarios al satisfacer rápidamente las necesidades financieras de los pacientes lesionados y sus familias después de una lesión, sin recurrir a un litigio.

Así pues, el cambio de paradigma de estos dos modelos supone un salto cualitativo en la gestión y resolución del error médico. Una mayor transparencia incrementa la confianza del paciente hacia el sistema sanitario y también hacia su médico. Dichas experiencias muestran que si se comunica el error, se piden disculpas y se ofrece una vía de reparación, ya no solo se disuade al paciente o sus familiares de tomar una vía más agresiva como sería la judicial, sino que en ocasiones no se reclama.

Estos modelos aportan beneficios en transparencia, autonomía del paciente, dignidad del profesional y seguridad clínica, por lo que sería recomendable seguir los mismos pasos. Ello supone, siguiendo al NHS, la implicación de la administración pública, la sanidad privada, colegios de médicos, asociaciones de pacientes y aseguradoras. Y por ello, como requisito indispensable para avanzar en esta dirección, se requiere un entorno de seguridad jurídica para los profesionales, lo que implica modificaciones legislativas en este sentido.

\section{BIBLIOGRAFÍA}

1. Kohn LT, Corrigan J, Donaldson MS. To err is human: building a safer health system. Washington, DC: National Academies Press 1999.

2. Arimany-Manso J, Martin-Fumadó C. La importancia de la seguridad clínica. Med Clin (Barc) 2017; 148: 405-407. https://doi. org/10.1016/j.medcli.2016.12.008

3. Martin-Fumadó C, Morlans M, Torralba F, ARImAny-Manso J. La comunicación del error médico. Consecuencias éticas y médicolegales. Med Clin (Barc) 2018. https://doi. org/10.1016/j.medcli.2018.07.013.

4. Gómez-Durán EL, Vizcaíno-RaKosnik M, MartinFumadó C, Klamburg J, Padrós-Selma J, ArimanyMANso J. Physicians as second victims after a malpractice claim: an importance issue in need og attention. J Healthc Qual Res 2018; 33: 284-289. https://doi.org/10.1016/j. jhqr.2018.06.002
5. Mira JJ, Carrillo I, Grupo SOBRINA. Medicina defensiva en Atención Primaria. An Sist Sanit Navar 2018; 41: 273-275.

6. Colegio Oficial de Médicos de Barcelona. Informe anual 2017. https://www.comb.cat/ cat/actualitat/publicacions/anuari/anuari2017/index.html. Consultado el 29 de noviembre de 2018.

7. Gómez-Durán EL, Martin-Fumadó C, ArimanyMANSO J. Legal medicine contributions to patient safety. From ascertainment and evaluation to research in medical liability. Int $\mathrm{J}$ Legal Med 2013; 127: 1051-1053.

8. Arimany-Manso J, Gómez-Durán EL. La gestión de la responsabilidad profesional médica desde el colectivo de la profesión médica y enfocada a la seguridad clínica. Rev Esp Med Legal 2013; 39: 142-148. https://doi. org/10.1016/j.reml.2013.09.001

9. Mira Solves JJ, Romeo Casabona C, Astier P, Urruela A, Carrillo I, Lorenzo S et al. Si ocurrió un evento adverso piense en decir "lo siento”. An Sist Sanit Navar 2017; 40: 279-290. https://doi.org/10.23938/ASSN.0031

10. Loren DJ, Garbutt J, Dunagan WC, Bommarito KM, Ebers AG, LEVINSON W et al. Risk managers, physicians, and disclosure of harmful medical errors. Jt Comm J Qual Patient Saf 2010; 36: 101-108. https://doi.org/10.1016/ S1553-7250(10)36018-1

11. Giraldo P, Corbella J, Rodrigo C, Comas M, Sala M, CASTElls X. Análisis de las barreras y oportunidades legales-éticas de la comunicación y disculpa de errores asistenciales en España. Gac Sanit 2016; 30: 117-120. https://doi. org/10.1016/j.gaceta.2015.11.007

12. NHS Resolution. Saying Sorry Guidance. https:// resolution.nhs.uk/wp-content/uploads/2017/07/ NHS-Resolution-Saying-Sorry-Final.pdf. Consultado el 29 de noviembre de 2018.

13. NHS Resolution. Delivering fair resolution and learning from harm. Our Strategy to 2022. https://resolution.nhs.uk/wp-content/ uploads/2017/04/NHS-Resolution-Our-strategy-to-2022-1.pdf. Consultado el 29 de noviembre de 2018.

14. NHS Resolution. Five years of cerebral palsy claims: A thematic review of NHS Resolution data. https://resolution.nhs.uk/wp-content/ uploads/2017/09/Five-years-of-cerebral-palsy-claims_A-thematic-review-of-NHS-Resolution-data.pdf. Consultado el 29 de noviembre de 2018 .

15. Massachusetts Alliance for Communication and Resolution following Medical Injury. www.macrmi.info. Consultado el 29 de noviembre de 2018 . 